\title{
Robust pole assignment techniques via state feedback
}

\author{
A. Varga \\ German Aerospace Center, DLR - Oberpfaffenhofen \\ Institute of Robotics and Mechatronics \\ D-82234 Wessling, Germany. \\ Andras. Varga@dlr.de
}

\begin{abstract}
We present a unifying computational framework to solve robust pole assignment problems for linear systems using state feedback. The new framework uses Sylvester equation based parametrizations of the pole assignment problems. The non-uniqueness of solutions is exploited by minimizing additionally sensitivity of closed-loop eigenvalues and the norm of the corresponding state feedback matrix. The solution methods rely on using gradient search based minimization techniques on suitably defined cost functions. The discussion of main functional and numerical aspects reveals many desirable features of the underlying algorithms which recommend them to serve as bases for robust numerical software implementations.
\end{abstract}

\section{Introduction}

Pole assignment techniques to modify the dynamic response of linear systems are among the most studied problems in modern control theory. The complete theoretical solution of this problem for standard systems has been followed by the development of many computational methods (see for example the collection of reprints in [13]). Sensitivity analysis of the pole assignment problem (see [10] and references therein) moves one step forward the understanding of difficulties and practical limitations associated with the usage of solution methods.

We address the eigenvalue assignment problem (EAP) for standard state-space systems of the form

$$
\lambda x(t)=A x(t)+B u(t),
$$

for descriptor systems of the form

$$
E \lambda x(t)=A x(t)+B u(t),
$$

and for linear periodic systems of the form

$$
x_{k+1}=A_{k} x_{k}+B_{k} u_{k} .
$$

In (1) and (2) $A, E \in \mathbb{R}^{n \times n}, B \in \mathbb{R}^{n \times m}$, and $\lambda x(t)=$ $\dot{x}(t)$ for a continuous-time system and $\lambda x(t)=x(t+1)$ for a discrete-time system. For the time-varying periodic system (3), $A_{k} \in \mathbb{R}^{n \times n}$ and $B_{k} \in \mathbb{R}^{n \times m}$ are periodic matrices with period $K \geq 1$. Let $\Gamma=\left\{\lambda_{1}, \ldots, \lambda_{n}\right\}$ be a given symmetric set of $n$ complex values.

We consider the following EAPs:

1. Standard eigenvalue assignment problem (SEAP): given the controllable pair $(A, B)$, determine the state feedback matrix $F \in \mathbb{R}^{m \times n}$ such that the eigenvalues of the closed-loop state matrix $A+B F$ are at desired locations $\Gamma$.

2. Descriptor eigenvalue assignment problem (DEAP): given the controllable descriptor pair $(A-\lambda E, B)$ and $\Gamma$ (possibly with infinite elements), determine the proportional-derivative feedback pair $(F, K)$ with $F, K \in \mathbb{R}^{m \times n}$ such that the generalized eigenvalues of the closed-loop descriptor pair $(A+B F, E+B F)$ are at desired locations $\Gamma$.

3. Periodic eigenvalue assignment problem (PEAP): given the completely reachable periodic matrix pair $\left(A_{k}, B_{k}\right)$, determine the periodic state feedback matrix $F_{k} \in \mathbb{R}^{m \times n}$ such that the eigenvalues of the closed-loop monodromy matrix $\Phi_{A+B F}(K, 0)=$ $\left(A_{K-1}+B_{K-1} F_{K-1}\right) \cdots\left(A_{0}+B_{0} F_{0}\right)$ are at desired locations $\Gamma$.

In the multi-input case or in the case $K>1$ for a periodic system the corresponding EAP has a non-unique solution. Therefore it is reasonable to exploit the nonuniqueness by imposing additional conditions. One aspect which is desirable from a practical point of view is to determine feedback matrices with small gains. Intuitively this must be advantageous since small feedback gains lead to smaller control signals, and thus to less energy consumption. Small gains are also beneficial to reduce noise amplification. A second aspect important in pole assignment is to achieve a small condition number for the eigenvector matrix of the state matrix or monodromy matrix of the closed-loop system. This is the goal of robust pole assignment $[7,4,14,6,9]$. In light of recent perturbation results for the SEAP [10], both aspects appear to be decisive for the low sensitivity of assigned eigenvalues. It was shown in [10] that 
high feedback gains or high condition numbers lead to high sensitivity of the closed-loop eigenvalues. Thus the simultaneous minimization of the feedback norm and condition of eigenvector matrix is a desirable general goal for solving EAPs.

In this paper we present a unifying framework for the numerically reliable solution of EAPs by exploiting the intrinsic non-uniqueness of the problems. The non-uniqueness issue is addressed by formulating the EAPs as minimum norm robust pole assignment problems $[15,16,17]$. By using Sylvester equations based parametrizations, those solutions of the EAPs are sought which minimize special cost functions expressing the weighted requirements for minimum norm feedback matrix and minimum sensitivity of closed-loop eigenvalues. The available explicit expressions for the gradients of cost functions allow the use of powerful gradient search based minimization techniques. The discussion of main functional and numerical aspects reveals many desirable features of the underlying algorithms which recommend them to serve as bases for robust numerical software implementations. Of particular importance is that all methods can address the eigenstructure assignment as well as partial pole assignment aspects.

\section{Parametrization of solutions}

\subsection{SEAP}

The parametrization is based on a straightforward Sylvester equation based formulation [3]. If $F$ solves the SEAP, then there exist $X$, an invertible transformation matrix, and $\widetilde{A}$ satisfying $\Lambda(\widetilde{A})=\Gamma$ such that

$$
X^{-1}(A+B F) X=\widetilde{A} .
$$

If we define $G:=F X$ then (4) can be rewritten as a Sylvester matrix equation

$$
A X-X \widetilde{A}+B G=0 .
$$

which must be satisfied by $X$.

Conversely, to solve the SEAP, we choose $\widetilde{A}$ such that $\Lambda(\widetilde{A})=\Gamma$, and assume that $G$ is a given parameter matrix. Then, we solve (5) for $X$ and, provided $X$ is invertible, we compute the feedback matrix as

$$
F=G X^{-1} .
$$

To enforce the invertibility of $X$, the matrices $\widetilde{A}$ and $G$ must fulfill standard conditions: 1$)$ the pair $(\widetilde{A}, G)$ is observable; 2) $\Lambda(A) \cap \Lambda(\widetilde{A})=\emptyset$. These conditions together with the controllability of pair $(A, B)$ ensure that $X$ satisfying (5) is generically invertible [5]. If $\widetilde{A}$ is in a Jordan canonical form, then the resulting $X$ plays the role of the eigenvector matrix for the closed-loop state matrix $A+B F$.

\subsection{DEAP}

If $F$ and $K$ are matrices which solve the DEAP, then there exist invertible matrices $X$ and $Y$ such that

$$
Y^{-1}(A+B F) X=\widetilde{A}, \quad Y^{-1}(E+B K) X=\widetilde{E},
$$

where the matrices $\widetilde{A}$ and $\widetilde{E}$ are such that $\Lambda(\widetilde{A}, \widetilde{E})=\Gamma$. If we define $G:=F X$ and $L:=K X$, then (7) can be rewritten as a Sylvester system of matrix equations

$$
\begin{aligned}
& A X-Y \widetilde{A}+B G=0 \\
& E X-Y \widetilde{E}+B L=0
\end{aligned}
$$

which must be satisfied by $X$ and $Y$.

Thus, to solve the DEAP, we choose $\widetilde{A}$ and $\widetilde{E}$ such that $\Lambda(\widetilde{A}, \widetilde{E})=\Gamma$, and assume $G$ and $L$ are given parameter matrices. Then, we solve (8) for $X$ and $Y$ and, provided $X$ and $Y$ are invertible, we compute the feedback matrices as

$$
F=G X^{-1}, \quad K=L X^{-1} .
$$

To enforce the invertibility of $X$ and $Y$, the matrices $\widetilde{A}, \widetilde{E}, G$ and $L$ must fulfill some standard conditions: 1) the pair $(\widetilde{A}-\lambda \widetilde{E}, G-\lambda L)$ is observable; 2$)$ $\Lambda(A, E) \cap \Lambda(\widetilde{A}, \widetilde{E})=\emptyset$. These conditions together with the controllability of pair $(A-\lambda E, B)$ ensure that $X$ and $Y$ satisfying (8) are generically invertible. If the pair $(\widetilde{A}, \widetilde{E})$ is chosen in a Weierstrass canonical form, then the resulting $X$ and $Y$ play the role of the closed-loop generalized eigenvector matrices for the closed-loop system pair $(A+B F, E+B K)$. Parametrizations of other two, more particular DEAPs, are considered in [16].

\subsection{PEAP}

The approach for solving SEAP can be extended to the more complicated case of PEAP. To simplify the presentation we will introduce some notation. For a square time-varying matrix $A_{k}, k=0,1, \ldots$, we denote $\Phi_{A}(j, i)=A_{j-1} A_{j-2} \cdots A_{i}$ for $j>i$ and $\Phi_{A}(i, i):=I$. If $A_{k}$ is periodic with period $K$, then the monodromy matrix of the system (3) at time $\tau$ is $\Phi_{A}(\tau+K, \tau)$. Its eigenvalues, denoted by $\Lambda\left(\Phi_{A}(\tau+K, \tau)\right)$, are independent of $\tau$ and are called characteristic multipliers. For an arbitrary periodic matrix $X_{k}$ of period $K$, also called a $K$-periodic matrix, we use alternatively the script notation $\mathcal{X}$ which associates the blockdiagonal matrix $\mathcal{X}=\operatorname{diag}\left(X_{0}, X_{1}, \ldots, X_{K-1}\right)$ to the cyclic sequence of matrices $X_{k}, k=0, \ldots, K-1$. This notation is consistent with the standard matrix operations like addition, multiplication, transposing, or inversion. Further we denote with $\sigma \mathcal{X}$ the $K$-cyclic shift $\sigma \mathcal{X}=\operatorname{diag}\left(X_{1}, \ldots, X_{K-1}, X_{0}\right)$ applied to the cyclic sequence $X_{k}, k=0, \ldots, K-1$.

To solve the PEAP we use a parametrization similar to the standard case. Let $G_{k} \in \mathbb{R}^{m \times n}$ be a given $K$-periodic parameter matrix and let $\widetilde{A}_{k} \in \mathbb{R}^{n \times n}$ be 
a given $K$-periodic matrix such that $\Lambda\left(\Phi_{\widetilde{A}}(K, 0)\right)=$ $\Gamma$. Consider the following periodic Sylvester equation (PSE)

$$
A_{k} X_{k}-X_{k+1} \widetilde{A}_{k}+B_{k} G_{k}=0, k=0, \ldots, K-1,
$$

and assume that its solution, the $K$-periodic matrix $X_{k}$, is invertible. Then, with the periodic feedback matrix

$$
F_{k}=G_{k} X_{k}^{-1}
$$

we have $X_{0}^{-1} \Phi_{A+B F}(K, 0) X_{0}=\Phi_{\widetilde{A}}(K, 0)$, and thus, $F_{k}$ solves the PEAP. Usual restrictions on choosing $\widetilde{A}_{k}$ and $G_{k}$ are similar to those in the standard case: (1) the periodic pair $\left(\widetilde{A}_{k}, G_{k}\right)$ is completely observable; and (2) $\Lambda\left(\Phi_{A}(K, 0)\right) \cap \Lambda\left(\Phi_{\widetilde{A}}(K, 0)\right)=\emptyset$. If additionally the periodic pair $\left(A_{k}, B_{k}\right)$ is completely reachable, then $X_{k}$ satisfying (10) is generically nonsingular and for the above $F_{k}$, we have $X_{k+1}^{-1}\left(A_{k}+B_{k} F_{k}\right) X_{k}=\widetilde{A}_{k}$. As in the standard case, $\widetilde{A}_{k}$ can be chosen to reflect the desired Jordan structure of the closed-loop monodromy matrix. For example, we can choose $\widetilde{A}_{0}$ in a real Jordan form and $\widetilde{A}_{k}, k=1, \ldots, K-1$, as identity matrices. In this case, each $X_{k}$ is the eigenvector matrix for the corresponding monodromy matrix $\Phi_{A+B F}(k+K, k)$.

\section{Optimization-based solution of robust EAPs}

In light of the sensitivity results in [10], it meaningful to exploit the non-uniqueness of the EAPs by computing solutions which ensure low sensitivity of the closed-loop eigenvalues and small feedback gains. This leads to a formulation of each EAP as a minimum norm robust $E A P$ for which the solution method relies on solving an unconstrained nonlinear programming problem. One of the main advantages of the Sylvester equation based parametrizations of EAPs is the possibility to easily derive explicit analytic expressions for the gradients of cost functions. This allows to employ efficient gradient based local search techniques to compute the "optimal" feedback by minimizing the underlying cost function.

Since the dimension of the minimization problems could be potentially large, a particularly well suited class of methods to solve our problems is the class of unconstrained descent methods, as for instance, the limited memory BFGS method [8] used in conjunction with a line search procedure with guaranteed decrease as that described in [12]. The guaranteed decrease feature of these methods ensures that the condition numbers of the solutions of Sylvester equations progressively decrease in each iteration and thus, these solutions remain invertible at each iteration once an invertible solution has been determined at the first iteration. In what follows, we define for each EAP the cost function to be minimized and give the corresponding result for the expressions of gradient.

\subsection{SEAP}

If $\widetilde{A}$ is in a Jordan form, then $X$ is the eigenvector matrix for the closed-loop system state matrix $A+B F$. As a measure of the sensitivity of closed-loop eigenvalues, we use the condition number $\kappa_{F}(X)$ of $X$ with respect to the Frobenius norm. For computational convenience, instead of minimizing $\kappa_{F}(X):=\|X\|_{F}\left\|X^{-1}\right\|_{F}$, the minimization of the sum $\|X\|_{F}^{2}+\left\|X^{-1}\right\|_{F}^{2}$ can be alternatively performed, since the two optimization problems are mathematically equivalent [4]. Thus, for the simultaneous minimization of the norm of the state feedback matrix $F$ and of the condition number $\kappa_{F}(X)$ we can use the following performance index

$$
J=\frac{\alpha}{2}\left(\|X\|_{F}^{2}+\left\|X^{-1}\right\|_{F}^{2}\right)+\frac{1-\alpha}{2}\|F\|_{F}^{2},
$$

where $0 \leq \alpha \leq 1$ is a weighting factor. For $\alpha=0 \mathrm{~J}$ defines a pure norm minimization problem, while for $\alpha=1$ we get a pure robust EAP. Intermediary values of $\alpha$ lead to a combination of both aspects.

The Sylvester equation based parametrization allows to derive explicit analytic expressions of gradients of the performance criterion $J$ with respect to the free parameter matrix $G[15]$.

Proposition 1 Let $F$ be the state feedback computed as in (6), assigning the desired eigenvalues $\Gamma$ for given $\widetilde{A}$ and $G$. Then, the gradient of $J$ with respect to $G$ is given by

$$
\nabla_{G} J=(1-\alpha) H^{T}+B^{T} U^{T},
$$

where $H=X^{-1} F^{T}$, and $U$ satisfies the Sylvester equation

$$
\widetilde{A} U-U A+S=0
$$

with $S=\alpha\left(-X^{T}+X^{-1} X^{-T} X^{-1}\right)+(1-\alpha) H F$.

Note that each function and gradient evaluation involves the solution of two Sylvester equations (5) and (14) sharing the same coefficient matrices.

\subsection{DEAP}

If the pair $(\widetilde{A}, \widetilde{E})$ is in a Weierstrass form then $X$ and $Y$ play the roles of the eigenvector matrices for the closedloop system pair $(A+B F, E+B K)$. For the simultaneous minimization of the norm of the state feedback matrices $F$ and $K$ and of the condition numbers $\kappa_{F}(X)$ and $\kappa_{F}(Y)$ we can use the following performance index

$$
\begin{aligned}
J= & \frac{\alpha}{2}\left(\|X\|_{F}^{2}+\left\|X^{-1}\right\|_{F}^{2}+\|Y\|_{F}^{2}+\left\|Y^{-1}\right\|_{F}^{2}\right) \\
& +\frac{1-\alpha}{2}\left(\|F\|_{F}^{2}+\|K\|_{F}^{2}\right),
\end{aligned}
$$

where $0 \leq \alpha \leq 1$ is a weighting factor, with the same meaning as in case of SEAP.

The following result gives the analytic expressions of gradients of the performance criterion $J$ with respect to the free parameters $G$ and $L$ for the DEAP [16]: 
Proposition 2 Let $(F, K)$ be the pair of state feedback matrices computed as in (9), assigning the desired eigenvalues $\Gamma$ for given $(\widetilde{A}, \widetilde{E})$ and $(G, L)$. Then, the gradients of $J$ with respect to $G$ and $L$ are given by

$$
\begin{aligned}
\nabla_{G} J & =(1-\alpha) F X^{-T}+B^{T} U \\
\nabla_{L} J & =(1-\alpha) K X^{-T}+B^{T} V
\end{aligned}
$$

where $U$ and $V$ satisfy the dual Sylvester system

$$
\begin{array}{rlr}
A^{T} U+E^{T} V & = & S \\
U \widetilde{A}^{T}+V \widetilde{E}^{T} & = & -T
\end{array}
$$

for

$$
\begin{aligned}
S= & (1-\alpha)\left(F^{T} F+K^{T} K\right) X^{-T} \\
& +\alpha\left(-X+X^{-T} X^{-1} X^{-T}\right) \\
T= & \alpha\left(-Y+Y^{-T} Y^{-1} Y^{-T}\right) .
\end{aligned}
$$

Note that each function and gradient evaluation involves the solution of a Sylvester system (8) and of a dual Sylvester system (17) sharing the same coefficient matrices.

\subsection{PEAP}

To solve the robust PEAP the condition numbers $\kappa_{F}\left(X_{k}\right)$ must be minimized [9]. This can be done simultaneously with the minimization of the norm of the feedback matrix. For this purpose we use the following weighted cost function to be minimized

$$
J=\frac{\alpha}{2} \sum_{k=0}^{K-1}\left(\left\|X_{k}\right\|_{F}^{2}+\left\|X_{k}^{-1}\right\|_{F}^{2}\right)+\frac{1-\alpha}{2} \sum_{k=0}^{K-1}\left\|F_{k}\right\|_{F}^{2} .
$$

The script notation allows to simplify the notation. $\mathcal{F}$ is computed as

$$
\mathcal{F}=\mathcal{G X}^{-1},
$$

where $\mathcal{X}$ satisfies the PSE

$$
\mathcal{A X}-\sigma \mathcal{X} \widetilde{\mathcal{A}}+\mathcal{B \mathcal { G }}=0 .
$$

Further $J$ can be expressed alternatively as

$$
J=\frac{\alpha}{2}\left(\|\mathcal{X}\|_{F}^{2}+\left\|\mathcal{X}^{-1}\right\|_{F}^{2}\right)+\frac{1-\alpha}{2}\|\mathcal{F}\|_{F}^{2} .
$$

With the above expression of $J$, we have the following result for the expression of the gradient [17]:

Proposition 3 Let $\mathcal{F}$ be the periodic feedback computed as in (18), assigning the desired characteristic values $\Gamma$ for given $\widetilde{\mathcal{A}}$ and $\mathcal{G}$. Then, the gradient of $J$ with respect to $\mathcal{G}$ is given by

$$
\nabla_{\mathcal{G}} J=(1-\alpha) \mathcal{H}^{T}+\mathcal{B}^{T} \mathcal{U}^{T},
$$

where $\mathcal{H}=\mathcal{X}^{-1} \mathcal{F}^{T}$, and $\mathcal{U}$ satisfies the PSE

$$
\widetilde{\mathcal{A}} \mathcal{U}-\sigma \mathcal{U} \sigma \mathcal{A}+\sigma \mathcal{S}=0
$$

with $\mathcal{S}=\alpha\left(-\mathcal{X}^{T}+\mathcal{X}^{-1} \mathcal{X}^{-T} \mathcal{X}^{-1}\right)+(1-\alpha) \mathcal{H} \mathcal{F}$.

Note that each function and gradient evaluation involves the solution of two PSEs (19) and (21) sharing the same coefficient matrices.

\section{Main algorithmic features}

Satisfactory eigenvalue assignment algorithms must fulfill several functional and numerical requirements to serve as bases for numerically robust software implementations. In what follows we discuss several algorithmic features of the Sylvester equation based approach and we point out how apparent limitations of this method can be overcome.

\subsection{Functional features}

Satisfactory computational algorithms must be general and flexible, and must exploit all structural aspects of the underlying problem. We will examine these aspects in case of the Sylvester equation based eigenvalue assignment methods.

\section{Generality}

Generality means that an eigenvalue assignment algorithm is able to assign an arbitrary set of eigenvalues, and ideally, it can also assign a desired eigenstructure for the closed-loop system. Although the first requirement seems to be trivial, even well-known methods implemented in commercial software are not able to fulfill this requirement. For example, the robust pole assignment method of [7] (implemented in function place of the MATLAB Control Toolbox) can not assign poles with multiplicities greater than rank of $B$ and the improved version of this approach has the same limitation [14]. The Sylvester equation based approach for the SEAP/DEAP/PEAP has no such limitations and can easily address the assignment of a given eigenstructure for the closed-loop system by suitably choosing the Jordan form of $\widetilde{A}$ for the SEAP, the Weiertrass form of the pair $(\widetilde{A}, \widetilde{E})$ for the DEAP or the periodic Jordan form of $\widetilde{\mathcal{A}}$ for the PEAP. The case of overlapping closed-loop and open-loop spectra can be also addressed by performing an initial eigenvalue shifting with a preliminary (random) feedback. For additonal details for SEAP and DEAP approaches, see [15, 16].

\section{Flexibility}

One apparent limitation of the Sylvester equation based approach is the need that the closed-loop and openloop spectra do not overlap. This condition guarantees the existence of a unique solution to the corresponding Sylvester equations (5), (8), or (19), and is thus convenient from numerical point of view when using Sylvester equation solvers. Although technical, this condition prevents the Sylvester equation based approach to perform a partial eigenvalue assignment, i.e., to keep unmodified some of the open-loop eigenvalues. Since the partial eigenvalue assignment is a very useful feature, especially when stabilizing high order systems, we show how this feature can be easily accommodated within the Sylvester equation based approach and thus substantially increasing its flexibility. 
In case of SEAP, the performance index $J$ in (12) is invariant to an orthogonal system similarity transformation, that is, if $F$ is the optimal feedback matrix for the pair $(A, B)$ then $\widehat{F}=F Q$ is the optimal feedback matrix for the transformed pair $(\widehat{A}, \widehat{B}):=\left(Q^{T} A Q, Q^{T} B\right)$, where $Q$ is an orthogonal matrix. Thus, if we want to keep unmodified the eigenvalues of $A$ lying in a "good" region $\mathbb{C}_{g}$ of $\mathbb{C}$ and to modify only those lying in its complement $\mathbb{C}_{b}=\mathbb{C} \backslash \mathbb{C}_{g}$ (the "bad" region), then we can first reduce $A$ to an ordered real Schur form (RSF) to obtain the pair

$$
Q^{T} A Q=\left[\begin{array}{cc}
A_{11} & A_{12} \\
0 & A_{22}
\end{array}\right], \quad Q^{T} B=\left[\begin{array}{c}
B_{1} \\
B_{2}
\end{array}\right]
$$

where $\Lambda\left(A_{11}\right) \subset \mathbb{C}_{g}$ and $\Lambda\left(A_{22}\right) \subset \mathbb{C}_{b}$. With this separation, we can perform a partial pole assignment by solving for the optimal solution $F_{2}$ the SEAP for the reduced pair $\left(A_{22}, B_{2}\right)$ and a corresponding reduced set $\Gamma_{2}=\Gamma \backslash \Lambda\left(A_{11}\right)$. The overall optimal feedback matrix results as $F=\left[\begin{array}{ll}0 & F_{2}\end{array}\right] Q^{T}$. A similar approach can also be employed in case of the PEAP (for details see [17]).

In case of DEAP, the performance index $J$ is invariant to an orthogonal system similarity transformation, that is, if $F$ and $K$ are the optimal feedback matrices for the descriptor pair $(A-\lambda E, B)$ then $\widehat{F}=F Z$ and $\widehat{K}=K Z$ are the optimal feedback matrices for the transformed pair $(\widehat{A}-\lambda \widehat{E}, \widehat{B}):=\left(Q^{T} A Z-\lambda Q^{T} E Z, Q^{T} B\right)$, where $Q$ and $Z$ are orthogonal matrices. Thus, if we want to keep unmodified the generalized eigenvalues of pair $(A, E)$ lying in $\mathbb{C}_{g}$ and to modify only those lying in its complement $\mathbb{C}_{b}$, then we can first reduce $(A, E)$ to an ordered generalized real Schur form (GRSF) to obtain the triple

$$
\begin{aligned}
Q^{T} A Z & =\left[\begin{array}{cc}
A_{11} & A_{12} \\
0 & A_{22}
\end{array}\right], \quad Q^{T} B=\left[\begin{array}{c}
B_{1} \\
B_{2}
\end{array}\right], \\
Q^{T} E Z & =\left[\begin{array}{cc}
E_{11} & E_{12} \\
0 & E_{22}
\end{array}\right],
\end{aligned}
$$

where $\Lambda\left(A_{11}, E_{11}\right) \subset \mathbb{C}_{g}$ and $\Lambda\left(A_{22}, E_{22}\right) \subset \mathbb{C}_{b}$. With this separation, we can perform a partial pole assignment by solving for the optimal solution $F_{2}$ and $K_{2}$ the DEAP for the reduced descriptor pair $\left(A_{22}-\lambda E_{22}, B_{2}\right)$. The overall optimal feedback matrices result as $F=$ $\left[\begin{array}{ll}0 & F_{2}\end{array}\right] Z^{T}$ and $K=\left[\begin{array}{ll}0 & K_{2}\end{array}\right] Z^{T}$.

\section{Structure exploitation}

The Sylvester equation approach in conjunction with the optimization based search for a minimum norm and well-conditioned feedback exploits the intrinsic freedom of the multi-input EAP to address an important additional requirement, namely, the well conditioning of the EAP. Note that most of pole assignment algorithms do not exploit this structural feature of the problem and even algorithms for robust pole assignment address only partially this aspect by ignoring norm minimization.
Moreover, most methods have also restrictions with respect to the allocation of the closed-loop eigenstructure.

\subsection{Numerical features}

We focus on discussing numerical properties like the numerical stability and computational efficiency of the Sylvester equation based eigenvalue assignment algorithms, and we address shortly the implementation aspects of this approach in robust numerical software.

\section{Numerical stability}

To solve each of the EAP, the computation of the optimal feedback for the computed optimal parameter matrix involves the solution of two systems of linear equations: the corresponding Sylvester equation (5), (8), or (19), and a linear system (e.g., $F X=G$ in case of SEAP to compute the feedback matrix $F$ ). Thus the Sylvester equation based approach can be considered to be practically numerically stable.

Concerning the accuracy of the results, in a robust pole assignment problem it is expected that the optimal $X$ or $\mathcal{X}$ (in case of PAEP) is reasonably well-conditioned, thus the last computational step is usually very accurate. Thus, the main source of errors appears to be the numerical solution of the Sylvester equations, where the separation of open-loop and closed-loop spectra is the essential factor for the accuracy of the computed $X$ or $\mathcal{X}$. However, a good separation can be always achieved by an initial eigenvalue shifting with a preliminary feedback, and therefore, for most practical problems, we can expect that the computed results corresponding to an optimal solution are very accurate.

\section{Efficiency}

The overall efficiency of the eigenvalue assignment algorithms heavily depends on the costs of function and gradient evaluations. Each function and gradient evaluation involves the solution of two Sylvester equations. In case of SEAP, the Sylvester equations (5) and (14) sharing the same coefficient matrices, can be solved using the Schur method [2]. This approach can be efficiently employed provided the matrix $A$ is reduced first to a RSF using an orthogonal similarity transformation and assuming further that the matrix $\widetilde{A}$ is in a Jordan form (a particular RSF with block-diagonal structure). The reduction of $A$, performed only once, can be seamlessly combined with the reordering of the RSF to accommodate with the partial pole assignment requirement. For the transformed problem, the function and gradient evaluations can be performed very efficiently since now we have to solve only reduced Sylvester equations with the coefficient matrices in RSF. The same technique can be employed to reduce the computational burden associated to evaluate the functions and gradients for the DEAP and PAEP. For details, see [16, 17]. 


\section{Implementation aspects}

The Sylvester equation based approach is simple to implement. For FORTRAN implementations to solve the SEAP and DEAP, all necessary software to perform the linear algebra computations is available in LAPACK 3.0 [1]. Here routines are provided to compute the RSF of a matrix, the GRSF of a matrix pair, to solve the Sylvester equation or Sylvester system, as well as systems of linear equations. For optimization, efficient unconstrained minimization routines are available in MINPACK-2 (the successor of MINPACK-1 [11]), offering a convenient reverse communication interface which allows an easy implementation of function and gradient computations.

For testing purposes, prototype MATLAB $m$-functions have been implemented by the author to solve the robust SEAP and several robust DEAPs (see [16]). These $m$-functions rely on two efficient mex-functions developed within the NICONET project ${ }^{1}$ : linmeq to solve various matrix equations and genleq to solve various generalized matrix equations. For optimization, the fminunc unconstrained minimization function available in the Optimization Toolbox 2.0 of MATLAB has been employed.

\section{Conclusions}

We surveyed recently developed reliable numerical approaches to exploit the intrinsic non-uniqueness of EAPs by formulating the EAP as a minimum norm robust pole assignment problem. The efficient evaluation of the cost functions and gradients is of paramount importance for the usefulness of the optimization based approach. Using transformation techniques in conjunction with the solution of reduced Sylvester equations is the main ingredient to achieve this goal. Further, it allows to address with practically no extra costs the partial pole assignment problem too. We believe that the presented robust pole assignment approaches are viable ways to solve large EAPs in the perspective of the requirements formulated by recent sensitivity analysis results [10]. Numerical results for the $\mathrm{SEAP} / \mathrm{DEAP} / \mathrm{PEAP}$ reported in $[15,16,17]$ indicate that the Sylvester approach produce the same or even better results than similar, but much more involved approaches $[14,6,9]$.

\section{References}

[1] E. Anderson, Z. Bai, J. Bishop, J. Demmel, J. Du Croz, A. Greenbaum, S. Hammarling, A. McKenney, S. Ostrouchov, and D. Sorensen. LAPACK User's Guide, Third Edition. SIAM, Philadelphia, 1999.
[2] R. H. Bartels and G. W. Stewart. Algorithm 432: Solution of the matrix equation $\mathrm{AX}+\mathrm{XB}=\mathrm{C}$. Comm. ACM, 15:820-826, 1972.

[3] S. P. Bhattacharyya and E. De Souza. Pole assignment via Sylvester's equation. Systems \& Control Lett., 1:261-263, 1922.

[4] R. Byers and S. G. Nash. Approaches to robust pole assignment. Int. J. Control, 49:97-117, 1989.

[5] E. De Souza and S. P. Bhattacharyya. Controllability, observability and the solution of $A X-X B=C$. Lin. Alg. \& Appl., 39:167-188, 1981.

[6] J. Kautsky, N. K. Nichols, and E.K.-W. Chu. Robust pole assignment in singular control systems. Lin. Alg. \& Appl., 121:9-37, 1989.

[7] J. Kautsky, N. K. Nichols, and P. Van Dooren. Robust pole assignment in linear state feedback. Int. J. Control, 41:1129-1155, 1985.

[8] D. C. Liu and J. Nocedal. On the limited memory BFGS method for large scale optimization. Mathematical Programming, 45:503-528, 1989.

[9] S. Longhi and R. Zulli. A note on robust pole assignment for periodic systems. IEEE Trans. Autom. Control, 41:1493-1497, 1996.

[10] V. L. Mehrmann and H. Xu. An analysis of the pole placement problem. II. The multi input case. Electronic Trans. Numer. Anal., 5, 1998. (http://etna.mcs.kent.edu/).

[11] J. J. Moré. User's Guide for MINPACK-1. Applied Mathematics Division Report ANL-80-74, Argonne National Laboratory, Argone,IL, 1980.

[12] J. J. Moré and D. J. Thuente. On line search algorithms with guaranteed sufficient decrease. ACM Trans. Math. Software, 20:286-307, 1994.

[13] R. V. Patel, A. J. Laub, and P. Van Dooren (Eds.). Numerical Linear Algebra Techniques for Systems and Control. IEEE Press, 1994.

[14] A. L. Tits and Y. Yang. Globally convergent algorithms for robust pole assignment by state feedback. IEEE Trans. Autom. Control, 41:1432-1452, 1996.

[15] A. Varga. Robust pole assignment via Sylvester equation based state feedback parametrization. In Proc. CACSD'2000 Symposium, Anchorage, Alaska, 2000.

[16] A. Varga. Robust pole assignment for descriptor systems. In Proc. MTNS'2000 Symposium, Perpignan, France, 2000.

[17] A. Varga. Robust and minimum norm pole assignement with periodic state feedback. IEEE Trans. Autom. Control, 45:1017-1022, 2000.

\footnotetext{
${ }^{1}$ see http://www.win.tue.nl/niconet/niconet.html
} 